PROCEEDINGS OF THE

AMERICAN MATHEMATICAL SOCIETY

Volume 140, Number 1, January 2012, Pages 227-233

S 0002-9939(2011)10886-4

Article electronically published on May 11, 2011

\title{
A PSEUDOROTATION WITH QUADRATIC IRRATIONAL ROTATION NUMBER
}

\author{
MARK TURPIN \\ (Communicated by Bryna Kra)
}

\begin{abstract}
We show, by explicit construction, that for any quadratic irrational number $\alpha$, there exists a pseudorotation on an indecomposable cofrontier $\Lambda$ with $\alpha$ as its rotation number. Our construction builds on a family of examples of Brechner, Guay, and Mayer. They observe that in the pseudorotations they construct, irrational numbers of constant type are not realized as rotation numbers. Circle rotations can realize any rotation number, but this is to our knowledge the first example of a pseudorotation with a quadratic irrational rotation number, and hence an irrational of constant type.
\end{abstract}

\section{INTRODUCTION AND DEFINITIONS}

We use the plane with the standard topology as the setting for the following mathematics.

A cofrontier $\Lambda$ in the plane is a compact, connected set that irreducibly separates the plane into exactly two components and is the boundary of each. For example, any topological circle is a cofrontier. The cofrontiers we consider are indecomposable; that is, they cannot be written as the union (not necessarily disjoint) of two compact, connected subsets. The two planar components, bounded and unbounded, can be conformally mapped to the open disk, and its exterior, respectively.

Any homeomorphism $f$ of the plane that leaves a cofrontier invariant will have on its bounded (or unbounded) component, a conjugate homeomorphism on the interior (or exterior) of the disk. These conjugacies are achieved via the abovementioned conformal map. All of these (interior and exterior) conjugate homeomorphisms have unique extensions to homeomorphisms on their boundary circles, and in general these circle homeomorphisms may be different. A pseudorotation on a cofrontier $\Lambda$ is a homeomorphism on $\Lambda$ that extends continuously to the plane, and for which the induced circle homeomorphisms, as described above, are both conjugate to the same rotation. Although pseudorotations as defined in BGM] include those whose cofrontiers are circles, our usage of the term pseudorotation will assume that the cofrontier is not a circle.

The rotation number $\alpha$ of a pseudorotation (or rotation) $f$ (restricted to $\Lambda$ ) may be thought of as limiting average angular progress, a notion made precise through consideration of the lift of a homeomorphism to a covering space. A circle rotation

Received by the editors October 18, 2010 and, in revised form, October 30, 2010 and November 5, 2010.

2010 Mathematics Subject Classification. Primary 37-XX; Secondary 11-XX, 26-XX, 54-XX, $58-\mathrm{XX}$.

(C)2011 American Mathematical Society 
by angle $2 \pi \alpha$ has rotation number $\alpha$. A homeomorphism $f \mid \Lambda$ has rotation number $\alpha$ if the limit $(n \rightarrow \infty) \Theta\left(f^{n}(x)\right) / n=2 \pi \alpha$ for all $x \in \Lambda$, where here the $\Theta$ is informal, being precisely defined via the lifted homeomorphism.

In 1981 Handel $[\mathrm{H}]$ constructed a diffeomorphic pseudorotation whose cofrontier is the pseudocircle, a highly indecomposable continuum. Handel's example is both minimal on the cofrontier, area-preserving on the plane, and infinitely differentiable. In 1991 Brechner, Guay, and Mayer [BGM] considered a broad class of pseudorotations, and among their many results proved that none of their examples has a rotation number which is an irrational of constant type, the class of irrational numbers that are the least well approximated by rational numbers. In 1996 the author showed [T] that in Handel's original example the rotation number is very well approximated by rationals. A general question is what set of irrational numbers are realizable as rotation numbers of pseudorotations? (All real numbers are realizable as rotation numbers of circle homeomorphisms.) In particular, we consider Question 6.1 from [BGM].

Question 6.1 ([BGM $)$. Does there exist an indecomposable cofrontier $\Lambda$ admitting an irrational pseudorotation for which the rotation number $\alpha$ is of constant type?

We show, by considering a construction outside the class considered in BGM], that every quadratic irrational number (a subset of the irrationals of constant type) is realizable as the rotation number of a pseudorotation.

Theorem. For any quadratic irrational number $\alpha$ there exists a pseudorotation on an indecomposable cofrontier $\Lambda$ with $\alpha$ as its rotation number.

\section{Elements of the COnstruction}

Our construction proceeds, up to a point, in the same manner as [BGM] from which we adopt our notation. We start with an annulus $A_{1}$ which we divide into an integer number, $q_{1}$, of links. Having chosen a forward direction, we define on $A_{1}$ a rigid rotation $g_{1}$ which advances each link to its adjacent link. We let $g_{1}^{m_{1}}$ be a rotation which advances $m_{1}$ links, where $m_{1}$ may be any integer such that $-q_{1}<m_{1}<q_{1}$. We can extend $g_{1}$ to all of $\mathbb{R}^{2}$ in many ways. For our purposes any continuous extension is sufficient. See Figure 1.

Next we embed a second annulus $A_{2}$ in $A_{1}$ with the requirement that $A_{2}$ be invariant under $g_{1}^{m_{1}}$ for all $m_{1}$. We require that $A_{2}$ be properly embedded in $A_{1}$ and that $A_{1}-A_{2}$ be a disjoint union of two topological annuli. Further, we define $g_{2}$ on $A_{2}$ to be a "rotation" by one link within the annular structure of $A_{2}$, again allowing $g_{2}^{m_{2}}$ as the possibility of advancing $m_{2}$ links in $A_{2}$. We let $f_{2}=g_{2}^{m_{2}} g_{1}^{m_{1}}$. See Figure 2.

Continuing inductively, given $A_{1}, A_{2}, \ldots, A_{k-1}$ we embed $A_{k}$ in $A_{k-1}\left(A_{k} \subset\right.$ $A_{k-1}$ where the inclusion is proper). Each annulus $A_{k}$ is divided into $q_{k}$ links on which we define a topological rotation $g_{k}$ which carries links to adjacent links. The rotation $g_{k}^{m_{k}}$ advances $m_{k}$ links within the annular structure of $A_{k}$. We define $f_{k}=g_{k}^{m_{k}} g_{k-1}^{m_{k-1}} \ldots g_{1}^{m_{1}}$ to be the composition of the first $k$ rotations and note that $A_{k}$ is invariant under $f_{k}$ (and $f_{k-1}, f_{k-2}$, etc.). We also note that $f_{k}$ can be extended continuously to all of $\mathbb{R}^{2}$. For a proof of the existence of this extension, see BGM].

Both the interior and exterior circle homeomorphisms induced by $f_{k}$ are rigid rotations with rotation number $\alpha_{k}=\sum_{i=1}^{k} \frac{m_{i}}{q_{i}}$. 


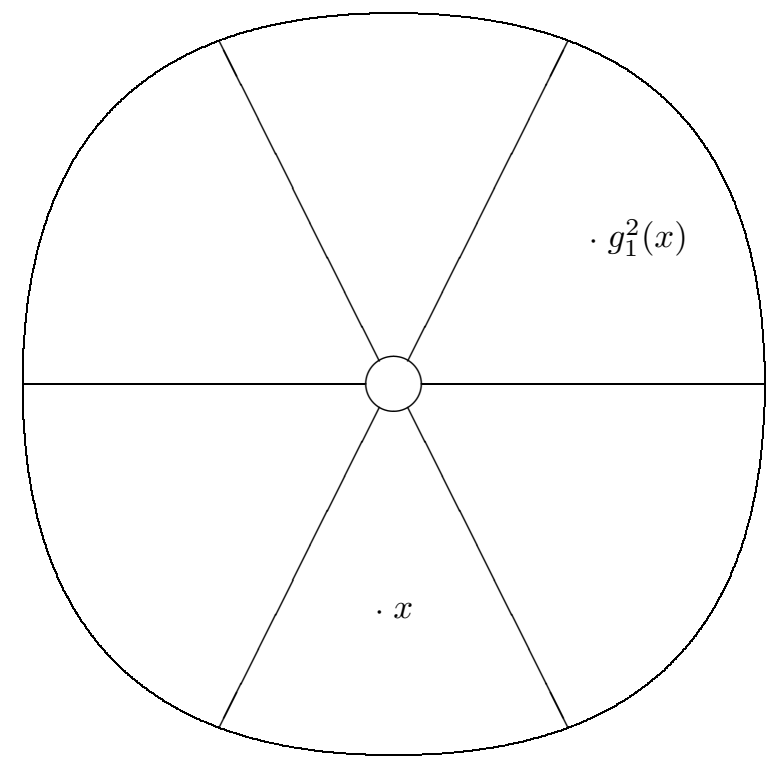

Figure 1 . An $A_{1}$ with 6 links, so $q_{1}=6 . f_{1}$ rotates by 2 links, so $m_{1}=2$.

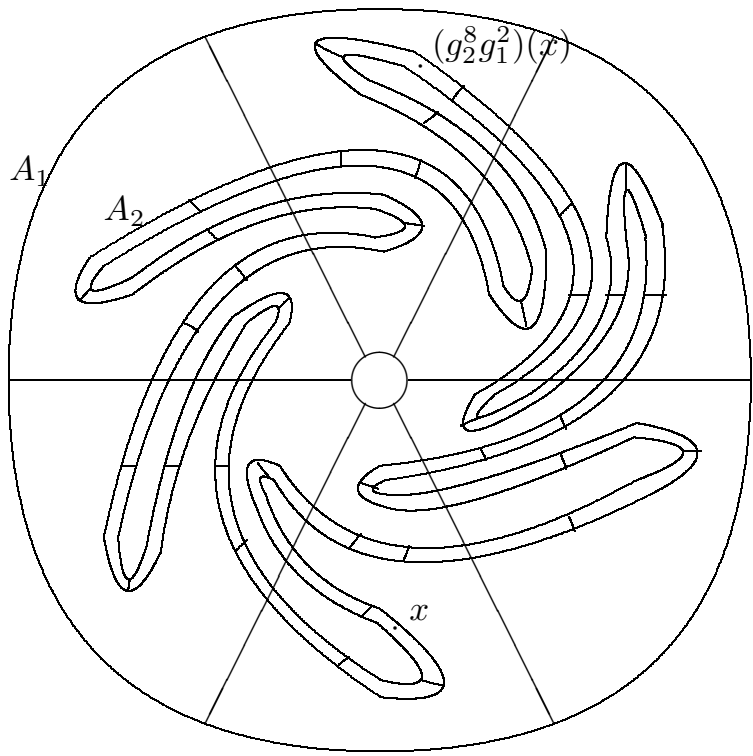

Figure 2. $A_{1}$ has 6 links $\left(q_{1}=6\right), A_{2}$ has 54 links $\left(q_{2}=54\right), f_{1}$ rotates by 2 links $\left(m_{1}=2\right)$ within $A_{1}$, and $f_{2}$ rotates by 8 links $\left(m_{2}=8\right)$ within $A_{2}$. 
We must show that the manner in which we embed these annuli guarantees that the cofrontier formed by the infinite intersection $\Lambda=\bigcap_{k=1}^{\infty} A_{k}$ is an indecomposable continuum.

We must also show that $\left\{f_{k}\right\}$ converges to a continuous function $f$ on $\mathbb{R}^{2}$, invariant on $\Lambda$, with rotation number $\alpha$ :

$$
\alpha=\lim _{k \rightarrow \infty} \alpha_{k}=\sum_{k=1}^{\infty} \frac{m_{k}}{q_{k}} .
$$

\section{The Rotation NUmber $\alpha$}

Let $\alpha$ be any quadratic irrational number. The irrationality of $\alpha$ implies that there exist infinitely many rational numbers $\frac{b}{c}$ such that

$$
\left|\alpha-\frac{b}{c}\right| \leq \frac{1}{c^{2}} .
$$

Among these rational numbers are all the convergents of $\alpha$, which are the rational numbers from each stage of the continued fraction expansion of $\alpha$. We denote these convergents by $\frac{b_{i}}{c_{i}}$. It is known that an irrational number is quadratic if and only if the terms of its (simple) continued fraction expansion are (eventually) periodic:

$$
\alpha=a_{1}+\frac{1}{a_{2}+\frac{1}{a_{3}+\ldots}}=\left[a_{1}, a_{2}, a_{3}, \ldots\right],
$$

where $\exists p, K \geq 1$ such that $\forall i \geq K, a_{i}=a_{i+p}$.

We examine the increasing sequence of denominators, $\left\{c_{i}\right\}$. We show that there exists a subsequence of denominators $\left\{c_{i_{k}}\right\}$ with the property $c_{i_{k}} / c_{i_{k-1}} \in \mathbb{Z}$.

Given $\alpha$, let $c_{i_{1}}$ be any denominator of any convergent of $\alpha$ with $i_{1}>K$. Let $q_{1}=c_{i_{1}}$. Now we wish to find $c_{i_{2}}$ which satisfies $c_{i_{2}} / c_{i_{1}} \in \mathbb{Z}$. We use the recurrence relation

$$
c_{i+1}=a_{i+1} c_{i}+c_{i-1},
$$

which determines the denominators of the convergents from the terms of the continued fraction expansion $\mathrm{N}$.

To find $c_{i_{2}}$ we use a subsequence of $\left\{a_{i}\right\}$, namely $\left\{a_{i_{1}+j p+1}\right\}, j \in \mathbb{Z}^{+}$. Because $p$ is the period of the sequence $\left\{a_{i}\right\}$, all terms in this subsequence are equal.

Consider the following triples of numbers:

$$
\left\{\left(a_{i_{1}+j p+1}, c_{i_{1}+j p}\left(\bmod c_{i_{1}}\right), c_{i_{1}+j p-1}\left(\bmod c_{i_{1}}\right)\right\}\right.
$$

The $a_{i+j p+1}$ are identical, ranging over $j$, and the other two terms are bounded by $c_{i_{1}}$. So for some value of $j$ we will see an ordered triple that is an exact repetition of an earlier triple. That is, $\exists j_{1}$ and $j_{2}\left(j_{2}>j_{1}\right)$ such that

$$
\begin{aligned}
& \left(a_{i_{1}+j_{1} p+1}, c_{i_{1}+j_{1} p}\left(\bmod c_{i_{1}}\right), c_{i_{1}+j_{1} p-1}\left(\bmod c_{i_{1}}\right)\right) \\
= & \left(a_{i_{1}+j_{2} p+1}, c_{i_{1}+j_{2} p}\left(\bmod c_{i_{1}}\right), c_{i_{1}+j_{2} p-1}\left(\bmod c_{i_{1}}\right)\right) .
\end{aligned}
$$

The equality of these terms and equation (10) implies that

$$
c_{i_{1}+j_{1} p+1} \equiv c_{i_{1}+j_{2} p+1}\left(\bmod c_{i_{1}}\right) \text {. }
$$

By rearranging terms of (1), we have

$$
c_{i-1}=a_{i+1} c_{i}-c_{i+1},
$$




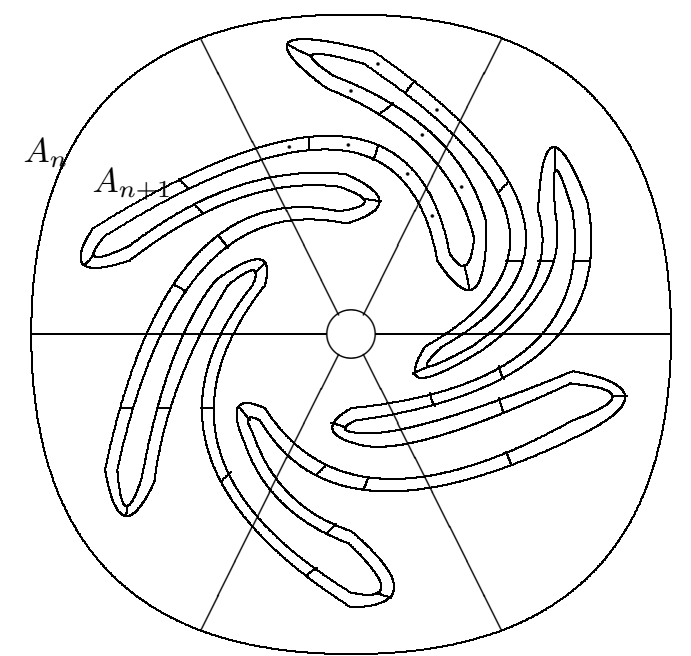

Figure 3. 9 links of $A_{n+1}$ form a basic repetitive unit within $A_{n}$.

which holds when equality is replaced with equality $\bmod c_{i}$. Hence

$$
\begin{gathered}
c_{i_{1}+j_{1} p-2} \equiv c_{i_{1}+j_{2} p-2}\left(\bmod c_{i_{1}}\right), \\
c_{i_{1}+j_{1} p-3} \equiv c_{i_{1}+j_{2} p-3}\left(\bmod c_{i_{1}}\right), \\
\ldots \\
c_{i_{1}} \equiv c_{i_{1}+\left(j_{2}-j_{1}\right) p}\left(\bmod c_{i_{1}}\right) .
\end{gathered}
$$

Similarly we can show

$$
c_{i_{1}} \equiv c_{i_{1}+\left(j_{2}-j_{1}\right) p} \equiv c_{i_{1}+2\left(j_{2}-j_{1}\right) p} \equiv c_{i_{1}+3\left(j_{2}-j_{1}\right) p} \equiv \ldots
$$

This gives us an unbounded sequence of denominators of convergents of $\alpha$ that are all multiples of $c_{i_{1}}$, and we will choose one to be $c_{i_{2}}$. We choose $c_{i_{2}}$ sufficiently large so that

$$
n_{2}=\frac{c_{i_{2}}}{c_{i_{1}}} \geq 4 c_{i_{1}}
$$

and we define $q_{2}=c_{i_{2}}$. So $q_{1}=c_{i_{1}}$ and $q_{k}=c_{i_{k}}$. Let $n_{k+1}=q_{k+1} / q_{k} \geq 4 q_{k}$. We can say that $n_{k+1}$ is the number of links in each basic repetitive unit of $A_{k+1}$. (A basic repetitive unit $B$ of $A_{n}$ is the smallest connected set of links of $A_{n}$ for which the union over $j$ of $g_{n-1}^{j}(B)=A_{n}$.) (See Figure 3.)

Because the fractions $b_{i} / c_{i}$ are convergents of $\alpha$, we can say

$$
\alpha=\frac{b_{i_{1}}}{c_{i_{1}}}+\left(\frac{b_{i_{2}}}{c_{i_{2}}}-\frac{b_{i_{1}}}{c_{i_{1}}}\right)+\left(\frac{b_{i_{3}}}{c_{i_{3}}}-\frac{b_{i_{2}}}{c_{i_{2}}}\right)+\ldots,
$$

and each term in the above sequence can be written as

$$
\frac{b_{i_{k+1}}}{c_{i_{k+1}}}-\frac{b_{i_{k}}}{c_{i_{k}}}=\frac{b_{i_{k+1}}-n_{k+1} b_{i_{k}}}{c_{i_{k+1}}} .
$$

Letting $m_{k+1}=b_{i_{k+1}}-n_{k+1} b_{i_{k}}$ and remembering $q_{k+1}=c_{i_{k+1}}$, we can write

$$
\alpha=\frac{m_{1}}{q_{1}}+\frac{m_{2}}{q_{2}}+\frac{m_{3}}{q_{3}}+\ldots
$$


Each finite sum is a convergent of $\alpha$ (but many convergents are excluded). Note that $m_{k}$ could be negative.

\section{Properties of the Construction}

We need to show that the $m_{k}$ and $q_{k}$ as defined satisfy two important properties:

(1) The cofrontier $\Lambda$, the intersection of the $A_{i}$, is indecomposable.

(2) The limit of the functions $f_{k}$ is a function.

We define $r_{2}$ to be the unique integer for which

$$
2 \leq r_{2} \leq \frac{n_{2}}{2 q_{1}}<r_{2}+1
$$

and similarly define $r_{k+1}$ as

$$
2 \leq r_{k+1} \leq \frac{n_{k+1}}{2 q_{k}}<r_{k+1}+1 .
$$

We require that each segment of $A_{k+1}$ that spans (crosses) a link of $A_{k}$ consists of at least $r_{k+1}$ links. Because each basic repetitive unit of $A_{k+1}$ has at least twice as many links as $A_{k}$, each basic repetitive unit of $A_{k+1}$ can span all of $A_{k}$. This condition is sufficient [CI for the indecomposability of the cofrontier $\Lambda$.

This condition also allows us to say that the diameter of each link of $A_{k+1}$ is less than half of the diameter of each link of $A_{k}$.

The irrationality of $\alpha$ gives the following for any convergent: $\left|\alpha-\frac{b_{i_{k}}}{c_{i_{k}}}\right|<\frac{1}{c_{i_{k}}^{2}}$.

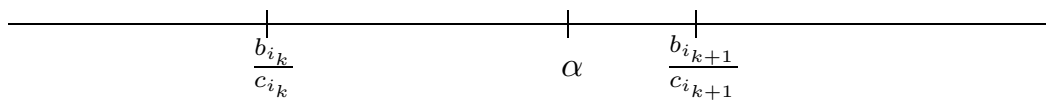

Figure 4. Convergents of alpha

We use this to say

$$
\left|\frac{m_{k+1}}{q_{k+1}}\right|=\left|\frac{b_{i_{k+1}}}{c_{i_{k+1}}}-\frac{b_{i_{k}}}{c_{i_{k}}}\right| \leq\left|\frac{b_{i_{k+1}}}{c_{i_{k+1}}}-\alpha\right|+\left|\alpha-\frac{b_{i_{k}}}{c_{i_{k}}}\right|<\frac{2}{c_{i_{k}}^{2}}=\frac{2}{q_{k}^{2}} .
$$

This implies that

$$
\left|m_{k+1}\right|<q_{k+1} \frac{2}{q_{k}^{2}}=n_{k+1} \frac{2}{q_{k}} .
$$

Combining with (2) and (3) from above we have

$$
\frac{\left|m_{k+1}\right|}{r_{k+1}}<n_{k+1} \frac{2}{q_{k}} \frac{1}{r_{k+1}}<\left(r_{k+1}+1\right) \frac{4}{r_{k+1}}=\left(1+\frac{1}{r_{k+1}}\right) 4<6 .
$$

So $g_{k+1}^{m_{k+1}}$ moves each link of $A_{k+1}$ through at most 6 links of $A_{k}$. The size of each link in $A_{k}$ is bounded by $(1 / 2)^{k}$, so the total distance that the composition $f_{k}$ "rotates" points is summable, and therefore $f_{k}$ converges to a continuous function $f$ on $\Lambda$ with a continuous extension to all of $\mathbb{R}^{2}$. 


\section{Questions}

Question 1. Given any irrational number $\alpha$, does there exist a pseudorotation on an indecomposable cofrontier with rotation number $\alpha$ ? In particular, are there irrational pseudorotations for irrationals of constant type that are not quadratic?

Question 2. Given a particular indecomposable cofrontier $\Lambda$, is there a way to characterize the set of rotation numbers of possible pseudorotations?

\section{REFERENCES}

[BGM] B. L. Brechner, M. D. Guay, and J. C. Mayer, The rotational dynamics of cofrontiers, Continuum Theory and Dynamical Systems (M. Brown, ed.), Contemporary Mathematics, vol. 117, American Math. Soc., 1991. MR 1112801

[CI] H. Cook and W. T. Ingram, A characterization of indecomposable compact continua, Proceedings of Topology Conference (held at Arizona State University, Tempe, AZ), Arizona State Univ., 1967. MR0253298(40:6513)

[H] M. Handel, A pathological area-preserving diffeomorphism of the plane, Proc. Amer. Math. Soc., 86, 1982. MR663889 (84f:58040)

[N] I. Niven, Irrational Numbers, The Carus Mathematical Monographs, no. 11, The Mathematical Association of America, distributed by John Wiley and Sons, Inc., 1956. MR0080123 (18:195c)

[T] M. Turpin, The restricted rotation number of an example of Handel, Dynamical Systems and Differential Equations, Vol. II (Springfield, MO, 1996), Discrete and Continuous Dynamical Systems, 1998. MR1722474 (2000g:37046)

Department of Mathematics, University of Hartford, West Hartford, Connecticut 06117 